\title{
A Differential Evolution-Based Approach for Global Localization of Mobile Robot
}

\author{
Minoru Ito ${ }^{\dagger}$ and Masahiro Tanaka ${ }^{\ddagger}$ \\ $\dagger$ Department of Control Engineering, Maizuru National College of Technology \\ 234 Aza Shiroya, Maizuru, Kyoto 625-8511, Japan \\ ¥ Department of Intelligence and Informatics, Konan University \\ 8-9-1 Okamoto, Higashinada-ku, Kobe 658-8501, Japan \\ E-mail: mito@maizuru-ct.ac.jp
}

\begin{abstract}
Mobile robot localization problem is determining a robot's pose in a given environment map. This problem is separated into three categories (position tracking problem, global localization problem, kidnapped problem) based on complexity. This paper presents a new evolutionary localization algorithm for global localization problem. Global localization problem aims to determine the robot's pose in a known environment without initial robot's pose information. In this study, we use differential evolution (DE) as evolutionary computation and define the global localization problem as a global optimization problem. Several studies have reported that differential evolution has an excellent ability for solving global optimization problems. The proposed method has been tested in some simulated environments to demonstrate the effectiveness.
\end{abstract}

Keywords: differential evolution, global localization, laser range sensor

\section{Introduction}

Mobile robot localization problem is the problem of determining the robot's pose (both its position and orientation) in a given environment map[1]. This problem is one of the most important problems towards the realization of an autonomous mobile robot[2].

Mobile robot localization problem is separated into three categories based on problem complexity[1]. The simplest localization problem is a "position tracking problem". In this problem the robot knows its initial pose. The goal of this problem is to keep track of the robot's pose while it's moving in the environment. The second problem is a "global localization problem". This problem is a more challenging task than the position tracking problem. In this problem the robot does not know its initial pose. The robot has to localize itself from scratch given the environment map and sensor data (e.g, laser range sensor data, web camera data). The third problem is a "kidnapped robot problem". This problem is a variant of the global localization prob- lem and is the most difficult problem. The complexity of this problem is to despite of the robot exactly knows pose itself, the robot is suddenly transferred to another location without aware of this.

For mobile robot localization problem, various methods have proposed[1]. In recent years, the probabilistic methods dominate the mobile robot localization algorithm. These methods are computable algorithm in real time and are based on Bayesian filter. In general, these methods are called Markov localization methods. Then these methods can be categorized in three families: Multi-hypothesis Kalman filters, grid-based Markov localization methods and Monte Carlo localization methods.

In recent years, applications of evolutionary computing are also studied as alternative methods for the mobile robot localization methods. Evolutionary computing can be used global optimum in practical time. This is one of well-known optimization algorithms outside of robotics. Evolutionary computing-based methods are similar to some Markov localization methods, where both of which adopt multipoint search algorithms. Because of this, some evolutionary computing have been applied to robotics. Duckett used genetic algorithm (GA) approach to solve simultaneous localization and mapping (SLAM) problem[3]. He applied GA to the mapping of the environment. Moreno et al. also used GA to solve the position tracking problem[4]. They applied GA to enhance Kalman filter in localizing the mobile robot. Kwok et al. used three evolutionary computing methods (GA, particle swarm optimization (PSO) and ant system (AS) ) to solve SLAM[5]. Moreno et al. used differential evolution (DE) to solve the global localization problem[6].

This paper present global localization method based on DE as with literature[6]. In our localization algorithm, we define the global localization problem as a global optimization problem. In our previous study, we applied DE to the position tracking problem[7] and tested the efficiency. In this study, we will test the efficiency of DE-based localization algorithm in the global localization problem.

The rest of this paper is organized as follows. Section 


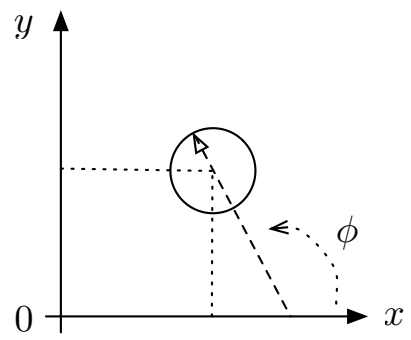

Fig. 1: Definition of the robot's pose in workspace.

2 describes the mobile robot global localization using differential evolution. Section 3 describes some experiments and results. Last section concludes this paper.

\section{Global Localization using Differential Evolution}

\subsection{Mobile Robot Global Localization}

Figure 1 shows the definition of the robot's pose in workspace. The robot's pose $\boldsymbol{x}_{r}(t)$ is defined by

$$
\boldsymbol{x}_{r}(t)=\left(x_{r}(t), y_{r}(t), \phi_{r}(t)\right)
$$

where $\left(x_{r}(t), y_{r}(t)\right)$ is position, $\phi_{r}(t)$ is orientation with respect to the $x$-axis and $t$ is time index.

Mobile robots recognize surrounding environment using some mounted sensor. In this study, we use one laser range scanner (e.g. Hokuyo URG-04LX, etc.) as a sensor. Sensor measures a distance to the surface of objects in the surrounding by evaluating the travel time of an emitted laser impulse. The observation data $\boldsymbol{z}(t)$ for one scan at time $t$ is defined by

$$
\boldsymbol{z}(t)=\left\{r_{\alpha}(t), \alpha=1, \ldots, N\right\}
$$

where $N$ is the amount of points in one scan.

The environment map data $m$ for mobile robot is defined by

$$
m=\left\{m_{\beta}, \beta=1, \ldots, N_{m a p}\right\}
$$

where $N_{\text {map }}$ is the amount of grids in environment map. In this study, we use grid-based map as an environment map.

In mobile robot global localization problem, mobile robot estimates own pose in the given environment map using observation data. Figure 2 shows the framework of mobile robot global localization.

\subsection{Differential Evolution}

Differential evolution (DE) is one of evolutionary computations for solving global optimization problem over continuous spaces. DE was proposed by Storn and Price in 1995[8]. Several studies have reported that DE has good performance in global optimization problem $[9,10]$. Moreover, some researches also show that DE can be applied in some real world problems,

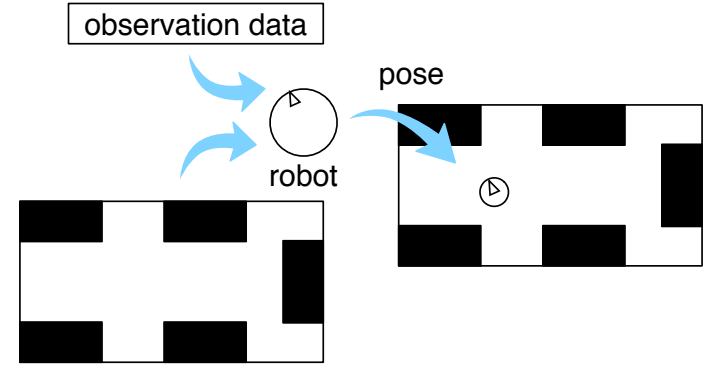

Fig. 2: Framework of mobile robot global localization.

such as multi objective optimization problems[11], dynamics optimization problems[12], design of digital IIR filters[13], image classification[14] and training of neural networks[15].

In the standard DE, an individual consists of $D$ dimensional vector. A population of $N P$ vectors is randomly generated at start of optimization task. The standard DE algorithm consists of three operators including mutation, crossover and selection operates. DE operators can be summarized as follows:

1. Mutation: For each target vector $\boldsymbol{x}_{i}(g)$, a mutated vector $\boldsymbol{v}_{i}(g)$ at generation $g$ is generated by

$$
\boldsymbol{v}_{i}(g)=\boldsymbol{x}_{p 1}(g)+F\left(\boldsymbol{x}_{p 2}(g)-\boldsymbol{x}_{p 3}(g)\right)
$$

where $i, p 1, p 2, p 3 \in[1,2, \ldots, N P]$ are randomly chosen and must be different from each other. Parameter $F$ is a scaling factor to control the differential vector $\left(\boldsymbol{x}_{p 2}(g)-\boldsymbol{x}_{p 3}(g)\right)$.

2. Crossover: The target vector $\boldsymbol{x}_{i}(g)$ is mixed with the mutated vector $\boldsymbol{v}_{i}(g)$ to produce trial vector $u_{i, j}(g+1)$

$$
u_{i, j}(g+1)=\left\{\begin{array}{lll}
\boldsymbol{v}_{i}(g) & \text { if } & r_{j}<C R \\
\boldsymbol{x}_{i}(g) & & \text { otherwise. }
\end{array}\right.
$$

where $j=1,2, \ldots, D$ denotes $j$-th parameter of individual vectors. $r_{j} \in[0,1]$ is the uniform random number. $C R$ is the crossover rate.

3. Selection: The trial vector $u_{i, j}(g+1)$ and its target vector $\boldsymbol{x}_{i}(g)$ are compared and the better one is selected by

$$
\boldsymbol{x}_{i}(g+1)=\left\{\begin{array}{l}
u_{i, j}(g+1) \text { if } \\
f\left(u_{i, j}(g+1)\right)<f\left(\boldsymbol{x}_{i}(g)\right) \\
\boldsymbol{x}_{i}(g) \quad \text { otherwise. }
\end{array}\right.
$$

where $\boldsymbol{x}_{i}(g+1)$ is the offspring of $\boldsymbol{x}_{i}(g)$ for the next generation. This selection scheme is usually named greedy selection.

In the DE algorithm, there are several strategies of mutation operators. The most useful strategies are :

- $\operatorname{rand} / 1$ :

$$
\boldsymbol{x}_{i}(g)=\boldsymbol{x}_{p 1}(g)+F\left(\boldsymbol{x}_{p 2}(g)-\boldsymbol{x}_{p 3}(g)\right)
$$


- best/1 :

$$
\boldsymbol{x}_{i}(g)=\boldsymbol{x}_{\text {best }}(g)+F\left(\boldsymbol{x}_{p 1}(g)-\boldsymbol{x}_{p 2}(g)\right)
$$

- current to best/1 :

$$
\begin{array}{r}
\boldsymbol{x}_{i}(g)=\boldsymbol{x}_{i}(g)+F\left(\boldsymbol{x}_{\text {best }}(g)-\boldsymbol{x}_{i}(g)\right) \\
+F\left(\boldsymbol{x}_{p 1}(g)-\boldsymbol{x}_{p 2}(g)\right)
\end{array}
$$

- best $/ 2$ :

$$
\begin{array}{r}
\boldsymbol{x}_{i}(g)=\boldsymbol{x}_{\text {best }}(g)+F\left(\boldsymbol{x}_{p 1}(g)-\boldsymbol{x}_{p 2}(g)\right) \\
+F\left(\boldsymbol{x}_{p 3}(g)-\boldsymbol{x}_{p 4}(g)\right)
\end{array}
$$

- $\operatorname{rand} / 2$ :

$$
\begin{array}{r}
\boldsymbol{x}_{i}(g)=\boldsymbol{x}_{p 1}(g)+F\left(\boldsymbol{x}_{p 2}(g)-\boldsymbol{x}_{p 3}(g)\right) \\
+F\left(\boldsymbol{x}_{p 4}(g)-\boldsymbol{x}_{p 5}(g)\right)
\end{array}
$$

where the indexes $p 1, p 2, p 3, p 4, p 5$ represent the random and mutually different integers generated and also different from index $i$. $\boldsymbol{x}_{\text {best }}$ is the best vector. In this paper, we adopt "best/1" as a mutation operator.

The main steps of the standard DE algorithm are given below:

1. Randomly generate a population of $N P$ vectors.

2. Evaluate the initial population. Calculate the objective function value $f\left(\boldsymbol{x}_{i}(g)\right)$.

3. Generate a new population.

(a) Randomly select 4 vectors $\boldsymbol{x}_{i}(g), \boldsymbol{x}_{p 1}(g)$, $\boldsymbol{x}_{p 2}(g), \boldsymbol{x}_{p 3}(g)$ from the population.

(b) Generate a mutated vector $\boldsymbol{v}_{i}(g)$ based on equation (4).

(c) Mix the target vector $\boldsymbol{x}_{i}(g)$ with the mutated vector $\boldsymbol{v}_{i}(g)$ based on equation (5).

(d) Evaluate the candidate vector $u_{i, j}(g+$ 1). Calculate the objective function value $f\left(u_{i, j}(g+1)\right)$.

(e) Adopt the candidate vector $u_{i, j}(g+1)$ in the new generation if it is better than current vector $\boldsymbol{x}_{i}(g)$.

4. Loop 3 until the termination criterion is met.

\subsection{Fitness Function}

If we try to deal with the global localization problem as an optimization problem, the natural choice for fitness function is the sum of squared error function. The fitness function is defined by

$$
f\left(\boldsymbol{x}_{r}(t)\right)=(\boldsymbol{z}(t)-\boldsymbol{z}(t))^{T}(\boldsymbol{z}(t)-\boldsymbol{z} \hat{(t)})
$$

where $\boldsymbol{z}(t)$ is the predicted observations according the estimated robot's pose at time $t$. \begin{tabular}{|l|l|l|}
\hline$x_{r}(t)$ & $y_{r}(t)$ & $\phi_{r}(t)$ \\
\hline
\end{tabular}

Fig. 3: Vector structure for DE.

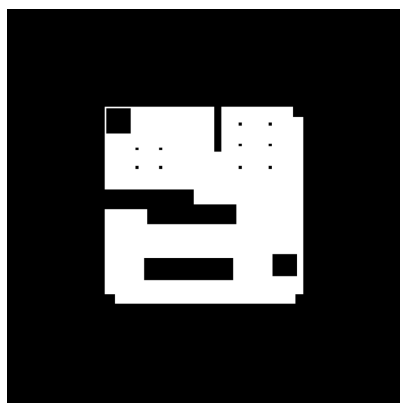

Fig. 4: Simulated environment 1.

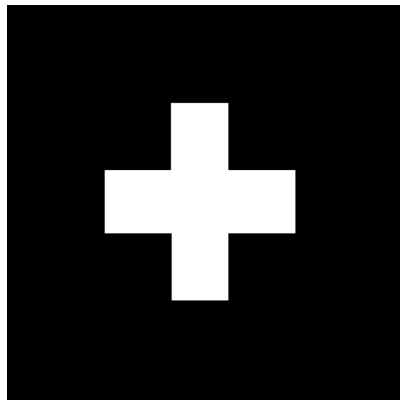

Fig. 5: Simulated environment 2.

Table 1: Parameter values for all experiments.

\begin{tabular}{|l|c|}
\hline Parameter & Value \\
\hline \hline DE algorithm & DE/best/1/exp \\
\hline population size & 400 \\
\hline generation & 200 \\
\hline parameter $F$ & 0.4 \\
\hline parameter $C R$ & 0.9 \\
\hline
\end{tabular}

\subsection{Implementation}

In this study, we define global localization problem as global minimization problem. Figure 3 shows the vector structure for DE. Each vector is encoded as a string of floating point numbers corresponding to the robot pose $\boldsymbol{x}_{r}(t)$. Each vector is evaluated by fitness function (eq.(12)). The global localization scheme is given below:

1. Give the environment map $m$ to the robot.

2. Get observation data $\boldsymbol{z}(0)$.

3. Optimization task by DE.

4. Output best result by step 3 . 
generation : 1

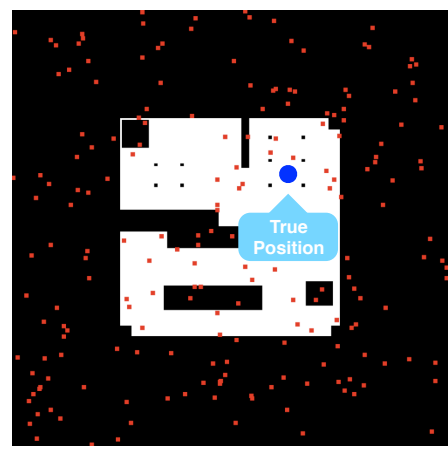

generation : 100

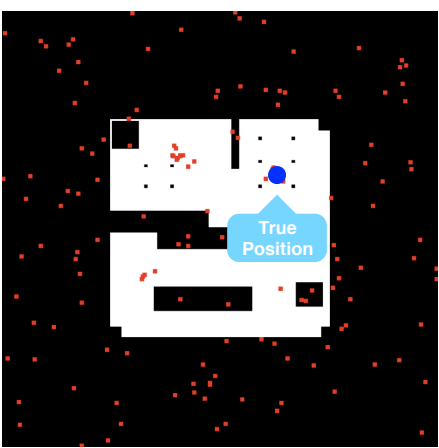

generation : 200

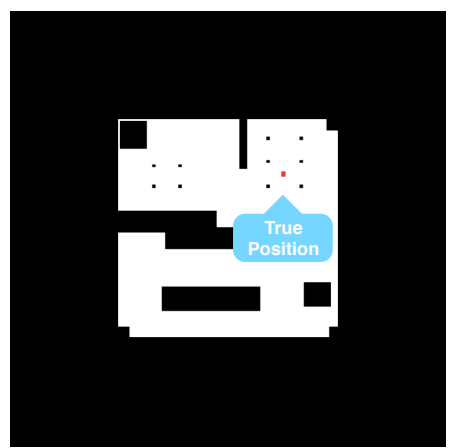

Fig. 6: Convergence of the proposed method in three different generation steps (Simulated environment 1).

generation : 1

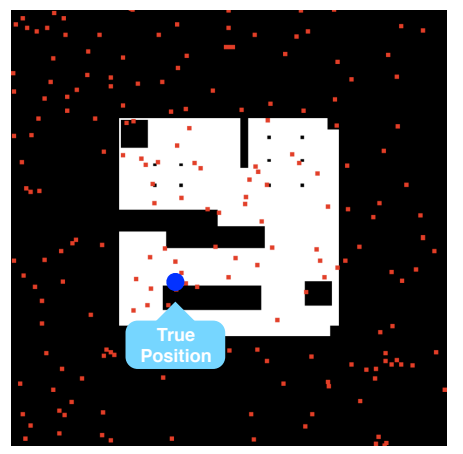

generation : 50

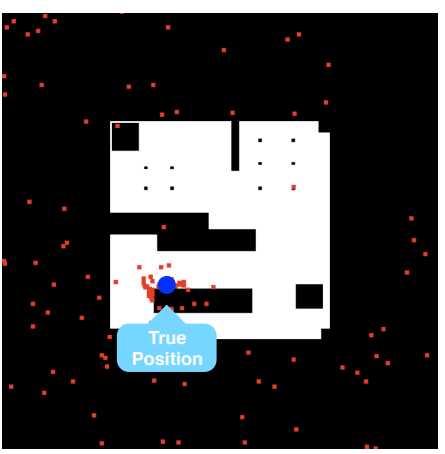

generation : 200

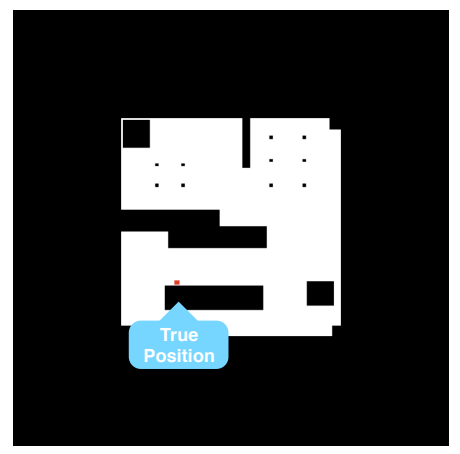

Fig. 7: Convergence of the proposed method in three different generation steps (Simulated environment 1).

\section{Experiments}

\subsection{Experimental Conditions}

In this study, we test the proposed method in two simulated environments. Figure 4 and Figure 5 show two simulated environments for all experiments. Each environment size is $20 m \times 20 m$ and is divided into $400 \times$ 400 grids. Each grid size is $50 \mathrm{~mm} \times 50 \mathrm{~mm}$. Simulated environment 1 is asymmetric environment. Simulated environment 2 is symmetric environment.

Table 1 shows parameter values for all experiments. In this study, we use "DE/best/1/exp" as DE strategy. The population size $N P$ is set to 400 and the number of generation is set to 200 . The scaling factor $F$ is set to 0.4 and the crossover rate $C R$ is set to 0.9 . All parameters are determined by trial-and-error.

\subsection{Experimental Results}

Let us discuss the simulation results at the robot's pose $\boldsymbol{x}_{r}(0)=\left(x_{r}(0), y_{r}(0), \phi_{r}(0)\right)=(2500,2500,60)$ in simulated environment 1 . Note that the mobile robot does not know own initial pose $\boldsymbol{x}(0)$. Figure 6 shows the distribution of the estimated robot's position (red points) in three different generation steps $(1,100,200)$. From this result, we can find out that candidate of the robot's pose converge on true robot's position. We also discuss the simulation results at the robot's pose $\boldsymbol{x}_{r}(0)=\left(x_{r}(0), y_{r}(0), \phi_{r}(0)\right)=(-2500,-2500,150)$ in simulated environment 1 . Figure 7 shows the distribution of the estimated robot's position (red points) in three different generation steps $(1,50,200)$. We can also find out that candidate of the robot's pose converge on true robot's position. The proposed method works well in an asymmetric environment like simulated environment 1.

Next, we discuss the simulation results in symmetric environment (simulated environment 2). In this experiment, the robot gets scan data at the robot's pose $\boldsymbol{x}_{r}(0)=\left(x_{r}(0), y_{r}(0), \phi_{r}(0)\right)=(0,-4000,90)$. Figure 8 shows the convergence of the estimated robot's position (red points) in five different generation steps $(1,5,10$, $15,20)$. In this case, we can find out that candidates of the robot's position converge on false robot's position. In this study, we assume the robot doesn't move. In symmetric environments, the robot can't estimate the true position by only one observation data. This is because the robot observes similar data from symmetry property of environments. In like this symmetric environment, global localization is very difficult problem. As solution for this problem, the robot should use multiple observation data for global localization. Using 
generation : 1

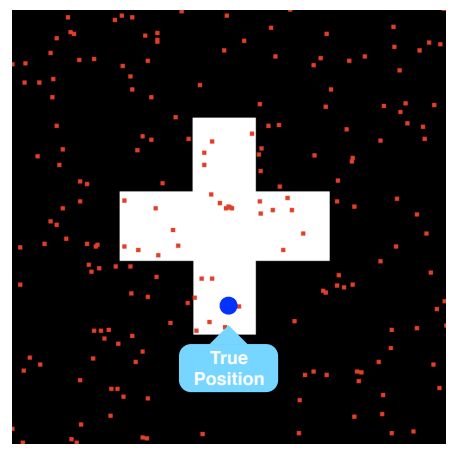

generation : 15

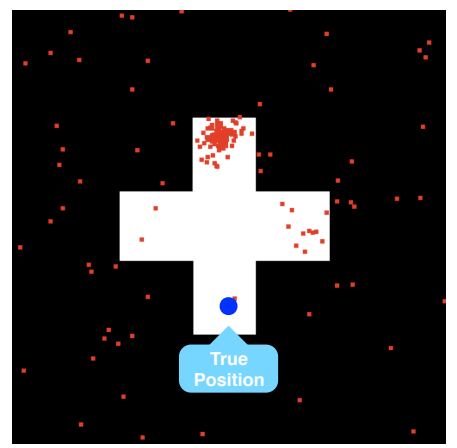

generation : 5

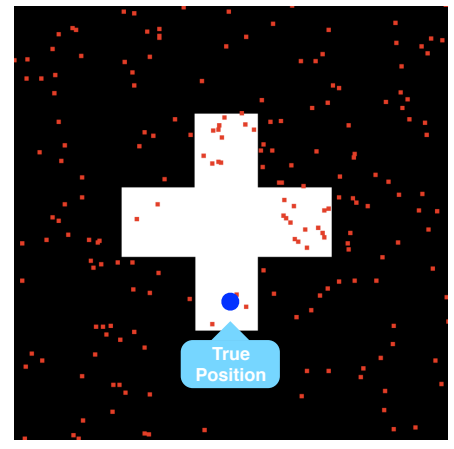

generation : 20

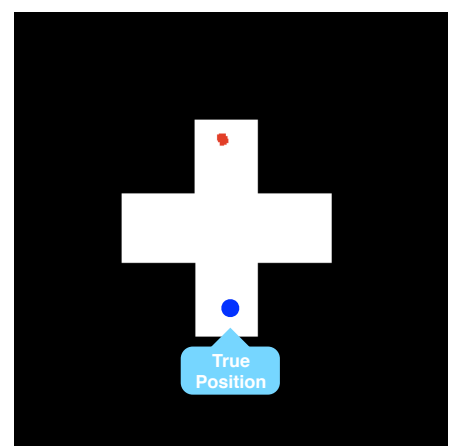

generation : 10

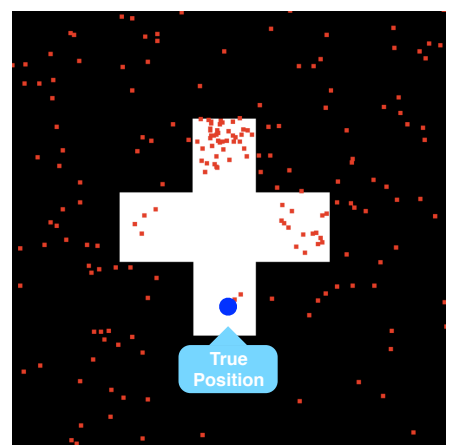

Fig. 8: Convergence of the proposed method in five different generation steps (Simulated environment 2).

multiple scan data, we can also improve accuracy of localization in asymmetric environments.

\section{Conclusions}

This paper presented new localization method using differential evolution for mobile robot global localization problem. We tested the proposed method using two simulated environments. Experimental results showed the efficiency of the proposed method and also showed some drawbacks.

In the future subjects, we will implement the proposed algorithm in an actual mobile robot system and make an experiment on-line. Additionally, we will make an experiment for comparing the proposed algorithm and other algorithms.

\section{Acknowledgment}

This research has been partially supported by "The Hirao Taro Foundation of the Konan University Association for Academic Research".

\section{References}

[1] S. Thrun, W. Burgard, and D. Fox : Probabilistic robotics, MIT Press, 2005.
[2] I. J. Cox: Blanche : Position estimation for an autonomous robot vehicle, Autonomous robot vehicles, 221/228, Springer-Verlag New York, Inc., 1990.

[3] T. Duckett : A genetic algorithm for simultaneous localization and mapping, Proceedings of the 2003 IEEE International Conference on Robotics and Automation, ICRA-2003, 434/439, 2003.

[4] L. Moreno, J. M. Armingol, S. Garrido, A. de la Escalera and M. A. Salichs : A genetic algorithm for mobile robot localization using ultrasonic sensors, Journal of Intelligent and Robotic Systems, Vol. 34, No. 2, 135/154, 2002.

[5] N. M. Kwok, D. K. Liua and G. Dissanayake : Evolutionary computing based mobile robot localization, Engineering Applications of Artificial Intelligence, Vol. 19, No. 8, 857/868, 2006.

[6] L. Moreno, S. Garrido and M. L. Munoz : Evolutionary filter for robust mobile robot localization, Robotics and Autonomous Systems, 54, 590/600, 2006.

[7] M. Ito and M. Tanaka : Mobile Robot Localization Using Differential Evolution, Proceedings of the 42th ISCIE International Symposium on Stochastic Systems Theory and Its Applications, 13/18, 2010.

[8] R. Storn and K. Price : Differential evolution - a simple and efficient adaptive scheme for global optimization over continuous spaces, Technical report TR-95-012, International Computer Science Institute, Berkley, 1995. 
[9] J. Vesterstrom, R. Thomsen : A comparative study of differential evolution, particle swarm optimization, and evolutionary algorithms on numerical benchmark problems , Proceedings of the 2004 congress on evolutionary computation, Vol. 2, 198/1987, 2004.

[10] R. Gamperle, S. D. Muller and P. Koumoutsakos : A parameter study for differential evolution, WSEAS International Conference on Advances in Intelligent Systems, Fuzzy Systems, Evolutionary Computation, 293/298, 2002.

[11] B. V. Babu, M. M. L. Jehan : Differential evolution for multi-objective optimization , Proceedings of 2003 Congress on Evolutionary Computation, 2696/2703, 2003.

[12] R. Mendes, A. S. Mohais : DynDE: a differential evolution for dynamic optimization problems , Proceedings of Congress on Evolutionary Computation, 2808/2815, 2005.

[13] N. Karaboga : Digital IIR filter design using differential evolution algorithm , EURASIP Journal on Applied Signal Processing, Volume 2005, Issue 8, 1269/1276, 2005.

[14] M. G. H. Omran, A. P. Engelbrecht and A.Salman : Differential evolution methods for unsupervised image classification, Proceedings of the 2005 IEEE Congress on Evolutionary Computation, 966/973, 2005.

[15] B. Subudhi and D. Jena : An improved differential evolution trained neural network scheme for nonlinear system identification, International Journal of Automation and Computing, Vol.6, No.2, 137/144, 2009. 